\title{
Report on the ECIR 2021 Discussion Panel on Open Access*
}

\author{
Djoerd Hiemstra \\ Radboud University, The Netherlands \\ hiemstra@cs.ru.nl
}

\begin{abstract}
On 31 March 2021, the Wednesday morning of ECIR 2021, the conference participants joined with seven panellists in a discussion on Open Access and Information Retrieval (IR), or more accurately, on the lack of open access publishing in IR. Discussion topics included the experience of researchers with open access in Africa; business models for open access, in particular how to run a sustainable open access conference like ECIR; open access plans at Springer, the BCS and the ACM; and finally, experience with open access publishing in related fields, notably in Computational Linguistics.
\end{abstract}

\section{Introduction}

Most publications in Information Retrieval - including the ECIR proceedings published by Springer on behalf of the BCS (British Computer Society), and the SIGIR proceedings published by the ACM - are not made available freely online by the publishers. To get access, university libraries or individuals have to pay subscription fees. As previously argued in SIGIR Forum by Hiemstra et al. [2020], these fees cannot be afforded by many universities in low-income countries, and there is a growing concern on how this impacts the inclusiveness of the field.

ECIR 2021 received 436 submissions from 488 authors from 40 countries. One continent is almost completely absent. Out of these 488 authors, only two authors were from Africa, one from North Africa (Egypt) and one from Sub-Saharan Africa (South Africa). IR conferences should reflect our world better. There is a great potential for the field to grow, if we can attract more researchers from Africa.

\section{The Panel}

A diverse panel of seven people involved in researching and publishing in IR shed their light on open access, including two representatives from Africa, one past ECIR general chair, and

*This report was previously published in BCS-IRSG Informer of Spring 2021. https://irsg.bcs.org/ informer/2021/04/ecir-2021-discussion-panel-on-open-access/ 
representatives from BSC IRSG, Springer, ACM SIGIR, and the Association for Computational Linguistics (ACL). Panellists were: Hassina Aliane (CERIST, Algeria), Ralf Gerstner (Springer Heidelberg, Germany), Min-Yen Kan (National University of Singapore), Haiming Liu (University of Bedfordshire, United Kingdom), Joao Magalhaes (Universidade Nova de Lisboa, Portugal), Hussein Suleman (University of Cape Town, South Africa), and Min Zhang (Tsinghua University, China).

Hassina Aliane, who is director of the Research and Development Division in Digital Humanities at the Research Centre for Scientific and Technical Information (CERIST) in Algeria, opened the discussion. Hassina explained that in Algeria, researchers have access to many subscription-based journals and proceedings by participating in a national program via CERIST. CERIST is the only government institution that provides researchers access. Hassina sees open access nevertheless as important. She is the editor of the open access journal Information Processing at the Digital Age that is published by the Algerian Scientific Journal Platform [ASJP, 2021] which facilitates open access journals in Algeria.

Hussein Suleman is Head of Department and Professor in Computer Science in the School of IT at the University of Cape Town in South Africa. Hussein mentioned that the University of Cape Town is relatively well-resourced, but that does not apply to most universities in SubSaharan countries, where no governmental research funding is available. Universities at those countries cannot afford the subscription fees of for instance Springer and the ACM, so access to IR publications is limited. Open Access would help researchers at those universities a lot. SubSaharan Africa's population is more than 4 times as big as the population of Northern Africa, but its share in African research is smaller than one may expect based on its population.

Joao Magalhaes is Associate Professor at the Department of Computer Science of the Universidade NOVA de Lisboa in Portugal. Joao was the General Chair of ECIR 2020 in Lisbon, the first successful on-line IR conference. Joao took the participants more than 20 years back to the web site of the IRSG in 1998 [IRSG, 1998] to show the importance of a central repository that holds the history of ECIR. Interestingly, the 1998 site was ahead of its time and for instance contained open access publications and even audio recordings of the talks of the Annual Colloquium on IR Research (the predecessor of ECIR). Joao took pride of the fact that the ECIR 2020 proceedings are open access at Springer. When ECIR 2020 went on-line because of the COVID-19 pandemic in Europe, costs of running the conference dropped substantially and about $€ 40,000$ from the registration fees were spent on open access article processing charges at $€ 30$ per page.

Ralf Gerstner is Executive Editor Computer Science at Springer-Verlag in Germany and among other things responsible for books in Information Retrieval, including the Information Retrieval Series and the LNCS proceedings (that include ECIR). Springer tries to meet the wishes of its customers for instance by agreements like Springer Compact that allow researchers from several countries to publish open access in Springer's journals, but not in the LNCS proceedings. Yet, LNCS generally allows for free access to the proceedings during the event, but this does not mean "real" open access, as they will be put behind a paywall again one to two weeks after the event. Additionally, individual authors or the conference organizers may pay for the effort to make 
the publications open access. Papers that are open access in Springer's hybrid proceedings and journals are download 8 to 10 times more often and cited 2 to 4 times more often. They also get more mentions on social media. Surprisingly, Ralf said that the ECIR 2020 proceedings are not open access at Springer (as no open access fee was paid) and that there must be some kind of misunderstanding with the ECIR 2020 organisers.

Haiming Liu is senior lecturer in Computer Science and Technology at the University of Bedfordshire in the UK, and elected member secretary of the BCS-IRSG. Haiming noted that, even though the BCS has an open access Electronic Workshops in Computing series [eWIC, 2021], the BCS IRSG chose for Springer about 20 years ago, because of the expected prestige and status of Springer's Lecture Notes in Computer Science for ECIR. ECIR is now a CORE A conference, and a transition to open access should not negatively impact its status.

Min Zhang is an associate professor at the Department of Computer Science at Tsinghua University Beijing in China, and the treasurer of ACM SIGIR. Min summarized the efforts that ACM SIGIR made the past years to support open access. This includes ACM's permanent access policies [ACM, 2021a]: Authorised links that enable free full-text downloads from the ACM Digital Library by so-called Open Table of Contents (OpenTOCs). Unfortunately, these OpenTOCs are no yet available for most SIGIR sponsored proceedings, even though SIGIR has requested this from the ACM. The ACM (as well as Springer) supports so-called "green open access", which means accepted preprints can be stored on institutional or preprint servers by the authors. It is yet unclear how ACM Open [ACM, 2021b] will impact the SIGIR conference and other sponsored conferences. ACM Open tries to make agreements between ACM and individual institutions, where institutions pay for the right of their researchers to publish open access at the ACM digital library. One concern is that in the future, researchers at universities that do not participate in ACM Open need to pay $\$ 700$ to $\$ 1700$ per accepted SIGIR paper.

Min-Yen Kan is an associate professor at the National University of Singapore. From 2008 until 2018, Min was the director of the ACL Anthology, the digital library of the Association for Computational Linguistics [ACL, 2021a], which is completely open access. The Anthology is funded by the ACL. The costs are minimal because the directorship and the volunteers are "human costs" that are recognized by the community, and web servers are donated by both the association as well as universities, such as the National University of Singapore. The Anthology is free and open source software [ACL, 2021b]. The ECIR or SIGIR community can fork and modify it to have its own IR Anthology if it chose to. This is not necessarily in competition with publishers, as one can enact a JSTOR-like moratorium if needed.

\section{Discussion and polls}

There was a lively discussion at the end of the panel, with questions ranging from Diversity, Equity and Inclusion to the possibilities for artefact reviewing and badging in an open access, volunteerled digital library. The discussion was finalised by three poll questions, to be answered by all participants (as well as the panellists): The first question, "Should ECIR move its proceedings 
to open access?" was answered with "yes" by $98 \%$ of the participants. The second question, "If ECIR were open access, what is the best solution?", was answered by $21 \%$ with "Springer", by $31 \%$ with the "BCS eWIC series", by $16 \%$ with "ACM Digital Library, and by $59 \%$ with "ACL Anthology", where participants could choose more than one answer. The third and final question, "How should we cover open access costs?" was answered by $21 \%$ by "BCS memberships", $65 \%$ answered by "Conference registration fees", 24\% answered by "Article processing charges", and another $65 \%$ answered "Using volunteer effort/human costs".

\section{Conclusion}

To conclude, an overwhelming percentage of $98 \%$ of the ECIR 2021 panel participants indicated that they want the ECIR proceedings to move to open access. Of the options discussed, a majority prefers the ACL Anthology, where costs are covered by the ECIR conference registration fees and volunteer effort.

\section{Acknowledgments}

I am grateful to Fabrizio Sebastiani, Raffaele Perego, and the ECIR conference volunteers for supporting the panel, and to Marie-Francine Moens for moderating questions via the chat.

\section{References}

ACL. Anthology. https://www.aclweb.org/anthology/, 2021a.

ACL. Github repository. https://github.com/acl-org/acl-anthology/, 2021b.

ACM. Permanent access. https://www.acm.org/publications/policies/permanent-access, 2021a.

ACM. Open: ACM's transformative model for open access publication. https://libraries. acm.org/subscriptions-access/acmopen, $2021 \mathrm{~b}$.

ASJP. Algerian scientific journal platform. http://www.asjp.cerist.dz/, 2021.

eWIC. Electronic workshops in computing. https://www.bcs.org/more/learned-publishing/ electronic-workshops-in-computing-ewic/, 2021.

Djoerd Hiemstra, Marie-Francine Moens, Raffaele Perego, and Fabrizio Sebastiani. Transitioning the information retrieval literature to a fully open access model. SIGIR Forum, 54(1), 2020.

IRSG. Site of the BCS Information Retrieval Specialist Group. https://web.archive.org/web/ 19980110084525/http://irsg.eu.org/, 1998. 